\title{
Vaginal partitioning with vertical septum-an alternative to colpocleisis
}

\author{
Sarita Kakatkar
}

Received: 21 October 2010 /Accepted: 8 November 2010 /Published online: 4 December 2010

(C) Springer-Verlag 2010

\begin{abstract}
The purpose of this study is to design and deploy an effective technique for prolapse repair in sexually inactive, geriatric patients which addresses all associated postoperative issues. A study group of 34 patients underwent vaginal partitioning with vertical septum (VPwVS). All 34 patients could successfully undergo VPwVS. Blood loss and surgical duration were comparable with conventional colpocleisis (CC). Postoperatively, no patient had recurrence of prolapse, de novo appearance, and/or recurrence of urinary or bowel symptoms, and no patient developed hematometra or pyometra. Pap smear collection is possible. This study shows that VPwVS is a holistic solution for prolapse repair among sexually inactive, geriatric patients, addressing all related issues and appears better than CC; however, a larger, multicentric study may be needed to statistically prove its advantage over CC. VPwVS's relevance is pronounced with an increasing geriatric population.
\end{abstract}

Keywords Colpocleisis · Prolapse repair · Vaginal partitioning

S. Kakatkar $(\square)$

Dr. Manohar Joshi Memorial Hospital (MJM Hospital), Janardan Sadan, 1194-23 Ghole Road,

Pune 411005, India

e-mail: sarita.kakatkar@gmail.com

e-mail: sarita.kakat@yahoo.co.in

\section{Background}

The Royal College of Obstetricians and Gynecologists recommends $\mathrm{CC}$ [1] for the repair of a prolapse in high-risk and sexually inactive, geriatric patients. Since its inception in 1876 or 1877 (Neugebauer-Le Fort), many modifications $[1,2]$ of colpocleisis have evolved to improve surgical outcome.

Core colpocleisis techniques address the symptoms of prolapse, but often are associated with urinary symptoms, which may need additional surgeries [3,4] varying from Kelly's stitch, pubocervical fascia plication, to TVT or mesh, all with variable success rates. Site-specific [5] repair for a cystocele and enterocele is also suggested to improve results. After colpocleisis, hematometra [2,6] and pyometra [7] have been reported. Pap smear collection is difficult. Rectal prolapse [8] and evisceration of the small bowel [9] were recorded after colpocleisis.

In view of the above, the goals of colpocleisis repair may be summed up as follows:

1. To relieve symptoms of prolapse with no recurrence [10-12]

2. To optimize bladder and bowel functions (no appearance of fresh urinary symptoms, enterocele, or rectal prolapse)

3. To permit natural drainage of uterovaginal secretions to prevent hematometra or pyometra

4. To permit Pap smear

The aforementioned goals need to be achieved without compromising the patient's medical condition and with minimal blood loss and surgical duration. 
The apex of the vagina is supported by fibromuscular tissue extending from one arcus tendineus to another, just below the vaginal epithelium [13], which should not get traumatized due to surgery, a risk inherent in the removal of vaginal mucosa. Creation of vertical septum, in contrast, does not involve the removal of vaginal epithelium, preventing possible trauma to the fibromuscular support arising from the arcus. Table 1 shows the comparison of CC with vaginal partitioning with vertical septum (VPwVS). It also contains Figs. 1 and 2.

\section{Introduction}

VPwVS described herein is an obliterative surgical procedure for prolapse repair, involving a departure from current textbooks and trends. This paper is qualitative in nature and belongs to the category "techniques and instrumentation." VPwVS is an obliterative procedure for prolapse repair involving partitioning of the vagina in bilateral tunnels, each of around $1.5 \mathrm{~cm}$ in diameter, (with or without hysterectomy) with a strong and broad, vertical, median

Table 1 Comparison of CC with VPwVS

Comparison of CC with VPwVS

$\mathrm{CC}$

VPwVS

\section{Basic concept}

Closure (partial or total) of vagina, usually with approximation of anterior and posterior wall

\section{Mucosa removal and vaginal shortening}

Raw area is created for approximation by removal of equal rectangular flaps of vaginal mucosa of anterior and posterior vaginal epithelium or circumcision of entire vaginal mucosa.

Mucosa removal and approximation causes vaginal shortening/closure.

\section{Risk and difficulty}

Dissection of vaginal mucosa of uniform thickness is difficult.

Vaginal mucosa removal carries the risk of damage to paracolpos or to lateral attachment of fibers which are attached to the pelvic bone.

\section{Septum/suture line}

The new suture line is transverse, hence directly exposed to abdominal pressure changes as shown in Fig. 1.

An under tension suture line may cause a high incidence of hematoma and its subsequent complications.

Additional purse-string sutures are needed before approximation if prolapse Baden-Walker grade is 3 or 4 .

\section{Plication of bladder neck and/or cystocele repair}

Kelly's stitch, TVT, urethral mesh, site-specific repair, etc. are needed to optimize bladder function.

\section{Supports}

No reinforcement of supports

Unequal support of urethra and bladder, disturbing UV angle

\section{Vaginal drainage and Pap smear}

Only a potential canal on either side of the suture line when uterus is conserved, no vaginal canal in total colpocleisis

Drainage possible; Pap smear difficult
Partitioning of vagina in bilateral tunnels with a vertical, median, and strong septum

There is no removal of vaginal mucosa. Redundancy is used to make a strong and broad septum.

Vertical, median septum is created extending to the vault, restoring the vaginal length.

Linear dissection of vaginal mucosa is easy.

There is no such risk since the mucosa is not removed. Further, even if the deeper supportive tissue gets traumatized, the outcome is not affected due to its integration with the septum while suturing.

The new suture line is vertical so the abdominal pressure changes are equally distributed on two neo vaginas and the vertical septum as shown in Fig. 2.

Tension-free suturing is possible, preventing hematoma and subsequent complications.

The procedure is the same irrespective of the Baden-Walker grading.

No additional procedure needed.

Indirect reinforcement of supports

Central defect of the bladder gets supported with a vertical septum. Lateral defects get corrected with inward turning of the vaginal mucosa, indirectly reinforcing bladder support. A small caliber of neo vaginas prevents prolapse too.

Equal support to urethra and bladder with no disturbance to UV angle

A 1.5-cm caliber canal on either side of the septum, neo vagina is lined by smooth vaginal epithelium all over as before, eliminating possibility of total closure

Neo vagina allows vaginal drainage, and Pap smear collection is possible. 
Fig. 1 Transverse section of vagina in $\mathrm{CC}$

along the vaginal length, which gives equal support to both anterior and posterior structures and also restores the vaginal length.

\section{Material and method}

This study is an ongoing project since 1995, conducted in non-teaching hospitals, undertaken as a variation of contemporary textbook versions of the obliterative procedures for prolapse repair, and informed consent was obtained from all the patients after a detailed discussion. This study consists of a total of 34 sexually inactive patients in the age group of 60 to 94 years, with a mean age of 77, complaining of prolapse. Figures 3 and 4 show the relevant information. While 31 patients were subjected to regional or general anesthesia, three patients were given local anesthesia+sedation. Figure 5 shows the type of surgery done in the study group.

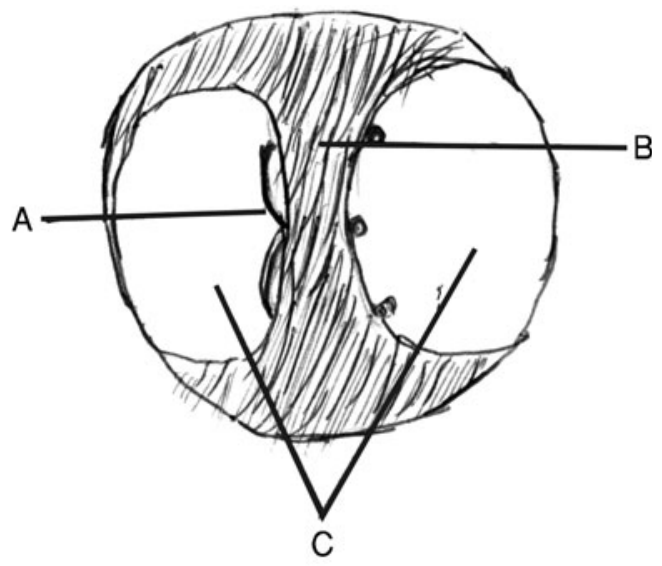

$$
\begin{aligned}
& A=\text { SUTURE LINE } \\
& B=\text { SEPTUM } \\
& C=\text { NEO VAGINA }
\end{aligned}
$$

Fig. 2 Transverse section of vagina in VPwVS

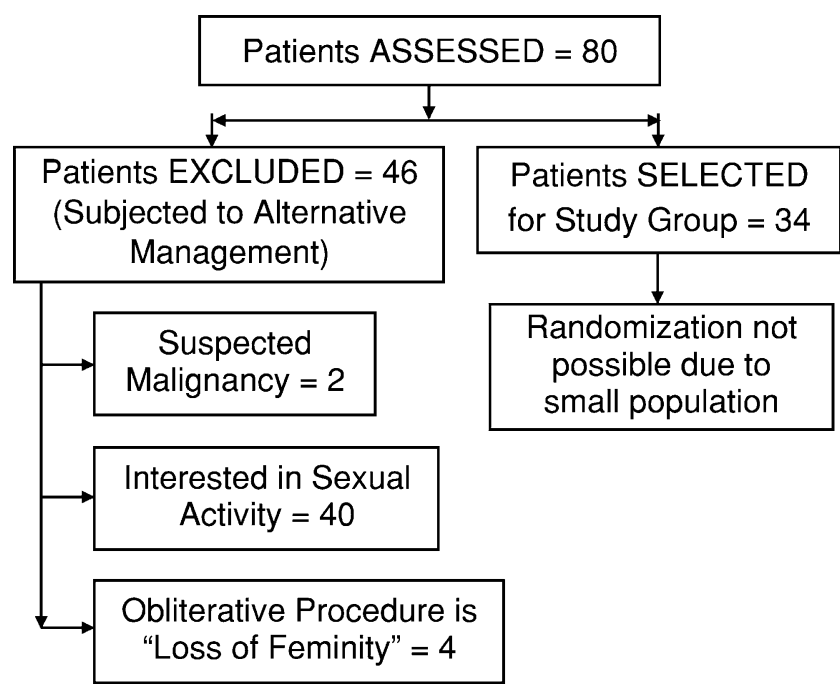

Fig. 3 Number of patients assessed and exclusion criteria

\section{Surgical steps in VPwVS}

The first step in VPwVS is to identify the vault before starting dissection. Saline+adrenaline (one in 100,000) is given submucosally. Aqua dissection helps to reduce intraoperative blood loss and possibility of trauma to nearby organs. The anterior wall is then dissected through a central, vertical incision anterior to the vault. Dissection should be $2 \mathrm{~cm}$ on either side of the incision to ensure tension-free suturing (see Fig. 6).

The posterior wall is dissected in two stages. The separation of the posterior wall is done at least $2 \mathrm{~cm}$ on either side of the central, vertical incision. The apex of the anterior incision is approximated with the apex of the posterior incision in the center of the vault with delayed absorbable sutures. The stitch passes exclusively through the raw area indirectly reinforcing the supports. This forms the apical point as shown in Fig. 7. While tightening the knot, ensure that the apical stitch is pushed as high as possible. Ensure that the suture length is equal on either side of the knot. Do not cut the suture material. The same suture material is used for the formation of the septum.

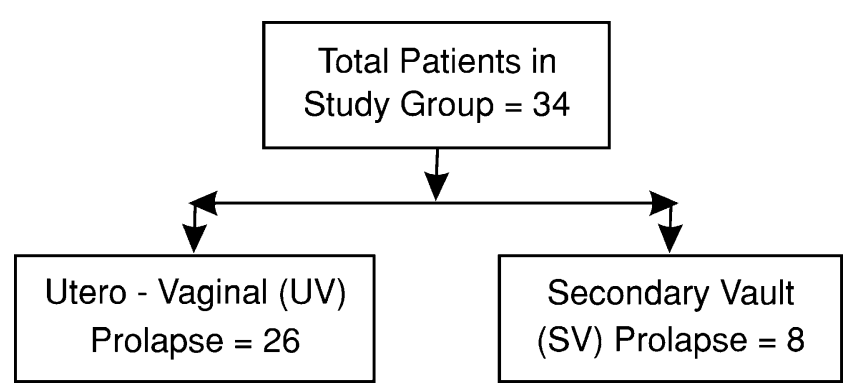

Fig. 4 Study group composition 


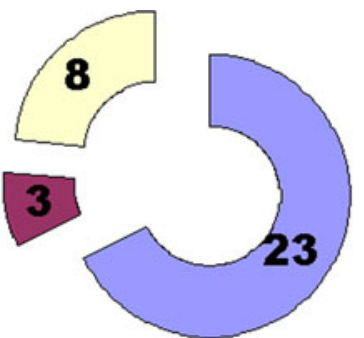

\section{$\square$ Hysterectomy followed by VPwVS (U-V Prolapse) $\square$ VPwVS without Hysterectomy (U-V Prolapse) $\square$ VPwVS alone (Secondary Vault Prolapse)}

Fig. 5 Type of surgery done in study group

For the formation of the septum, continuous vertical mattress stitches are taken starting from the apical point (point e in Fig. 8). One end of the suture is used to make stitches on the right side while the other end is used for suturing on the left side. To start with, one end of the suture is passed through a point $1.5 \mathrm{~cm}$ above the apical point and at the base of the right anterior wall (point a). The needle enters from the raw area near the apical point to the shiny vaginal mucosa. To complete the vertical mattress stitch, the needle is inserted at a point $1.5 \mathrm{~cm}$ above point ' $\mathrm{a}$ ' at the base of the right anterior wall (point $b$ ). The needle comes from the vaginal mucosal side in the raw area. The same end of the suture material is used to complete the vertical mattress stitch on the right posterior wall. To start with, the needle is inserted at a point $1.5 \mathrm{~cm}$ below the apical point (point c) at the base of the right posterior wall from the raw area to the vaginal mucosa. To complete the vertical mattress stitch, the needle is now reinserted through a point $1.5 \mathrm{~cm}$ below point 'c' (point d) at the base of the right posterior wall. The suture material comes from the vaginal mucosal side to the raw

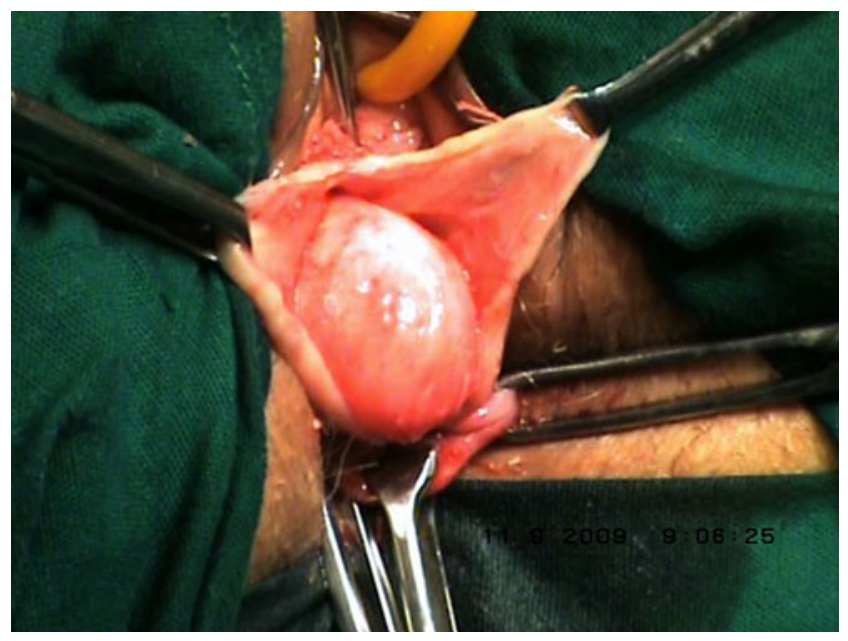

Fig. 6 Anterior wall dissection

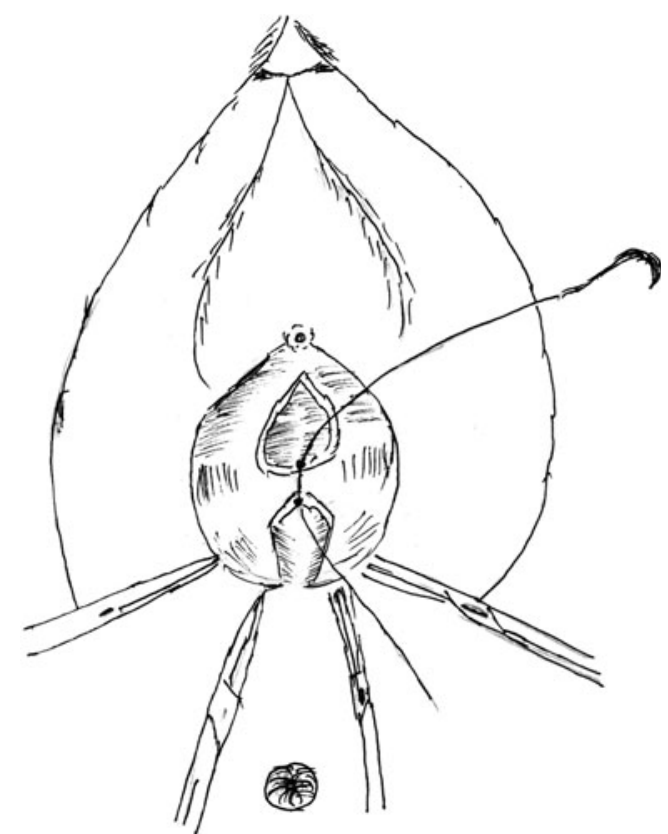

Fig. 7 Apical point

area. The other end of the suture is taken through the left anterior and posterior vaginal wall in a similar way.

After the sutures are completed on either side, traction is given to them with the result that the edges are out-turned in the lumen on each side. The knot is then tied firmly pushing up the apical point. The knots are at the center, covered by the raw dissected area. This helps to make a vertical septum dividing the vagina longitudinally in two narrow tunnels. The dissected raw area gets plastered with

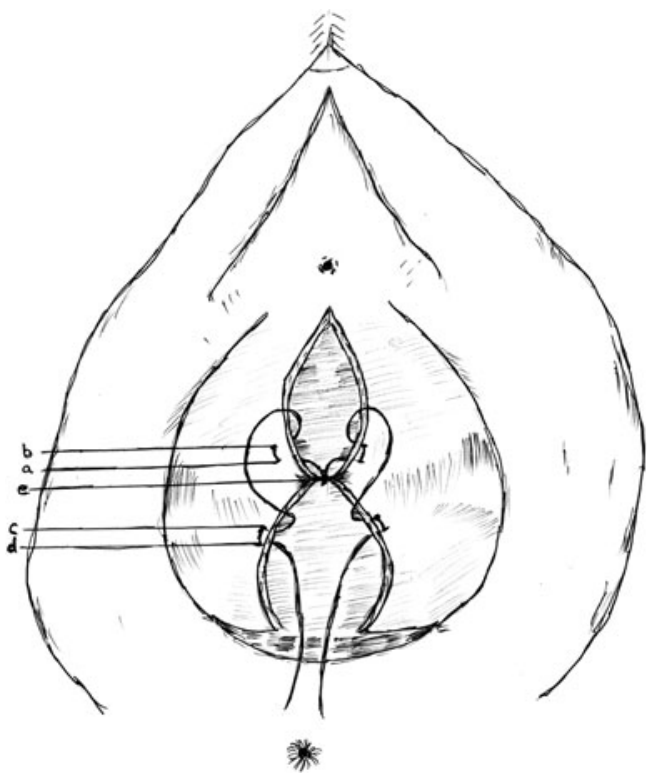

Fig. 8 Suturing 
each other creating a broad and strong septum. Similar vertical mattress stitches are taken along the total length of the anterior wall incision.

Since the posterior wall is always longer than the anterior wall, a portion of the posterior wall remains not taken up. A wedge-shaped portion is now removed on either side of the median split, and a routine colpoperineorrhaphy is done after approximation of the levator ani followed by the closure of the perineal skin.

A double vagina is created (points $a$ and $b$ in Fig. 9) with a central septum (point $\mathrm{c}$ ) extending from the introitus to the apical point, restoring the vaginal length. The caliber of each half of the double vagina is around $1.5 \mathrm{~cm}$.

\section{Findings}

The mean surgical duration was 45 min while the mean blood loss was 35 milliliters, with a $p$ value of zero in both cases. Both are comparable in VPwVS and CC [14].

The first follow-up (FU) was after 6 weeks. Subsequent follow-ups were done on a 3-month basis for a period of 1 year and annually thereafter (see Fig. 10 Postoperative Follow-Up : AFTER ONE YEAR). All patients were screened postoperatively for recurrence of prolapse, urinary symptoms, and other complaints, if any. No patient had recurrence of prolapse, stress incontinence, or rectocele (calculated $p$ value $=0$ ).

Surgical duration and blood loss in VPwVS compare with those of CC [14]. The number of patients lost for FU in this study (Fig. 10), though apparently considerable, compares with CC [15], but none of the patients in this study had recurrence, urinary symptoms, or rectal prolapse.

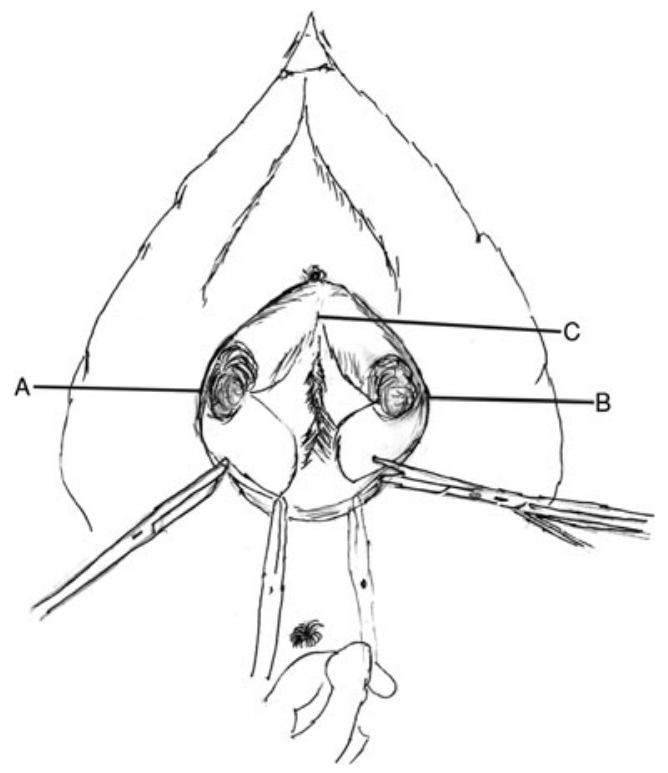

Fig. 9 End result

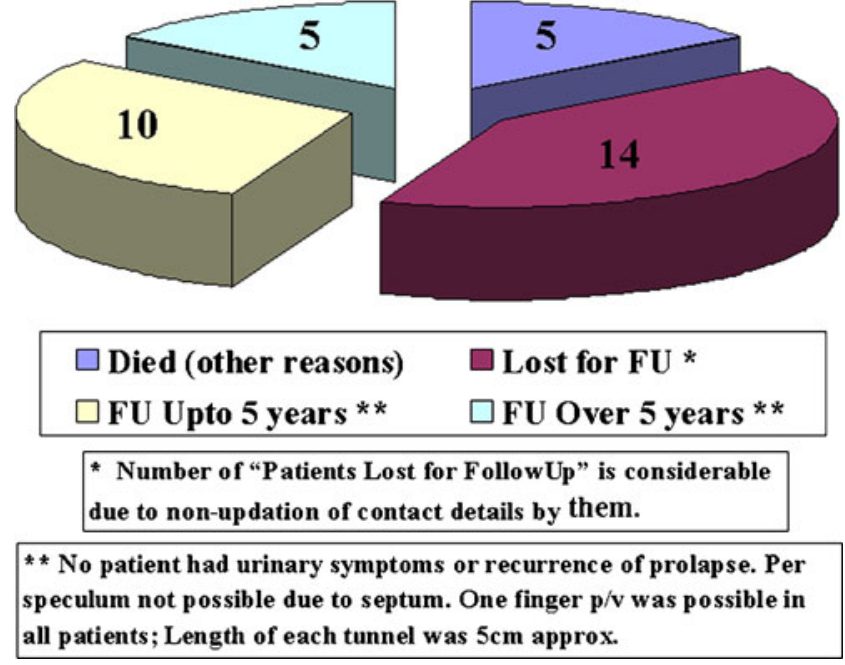

Fig. 10 Postoperative follow-up after 1 year

As shown in Table 1, the VPwVS procedure appears to have an advantage over $\mathrm{CC}$ because of the following:

- More anatomical and less distortive

- Independent of extent or site of prolapse

- Needs no associated surgery, thus saving on surgical duration and blood loss

- Safe, simple, and elegant to perform

- Effective and gives long lasting results

VPwVS is also feasible within the given constraints, i.e., without compromising the patient's medical condition and with minimum surgical duration and blood loss.

\section{Conclusion}

Geriatric population is on the rise and so is the incidence of prolapse. The role of obliterative surgery for prolapse repair is now well accepted in geriatric patients disinterested in coital activity. Such obliterative procedures need to be effective, with lasting results, and also need to be safe and simple. VPwVS achieves all the goals of colpocleisis with good success rate, even on long-term follow-up; however, a larger, multicentric study may provide a statistical comparison between VPwVS and CC. Being technically and functionally sound, VPwVS is a definite alternative to colpocleisis and merits larger deployment by the professionals.

Acknowledgements Dr. Tushar Panchnadikar, M.D. (ObGy), a professor from Bharati Vidyapith Medical College, Pune; Dr. Anjali Radkar, Ph.D. (statistics), an associate professor at the Gokhale Institute of Economics, Pune; and Mr. Pradeep Kakatkar, FCA, Pune are gratefully acknowledged.

Details of ethics approval Guidelines prescribed in the Medical Ethics Manual (2009) of WMA are fully complied with. 


\section{References}

1. RCOG/BSUG (British Society of UroGynaecology) (2007) Management of post-hysterectomy vaginal vault prolapse: greentop guideline \#46. http://www.rcog.uk/..../Guidelines. Accessed Dec-2009 to Jun-2010

2. Goldman J, Ovadia J, Felfberg D (1981) The Neugebauer-Le Fort operation: a review of 118 partial colpocleisis. Eur J Obstet Gynecol Reprod Biol 12:31-35, http://www.jfponline.com/pagesasp?aid=7597.

3. Hubbard L, et al (2005) Vaginectomy right for some with pelvic prolapse. www.findarticles.com/p/article/mi_mocyd/is_22_39/ ai n8568368. Accessed Dec-2009 to Jun-2010

4. Harmanli $\mathrm{OH}$ (2002) Largest case series to date: colpocleisis seen as effective for prolapse in elderly. Ob Gyn News 37(8):18, www. jfponline.com/pagesasp?aid $=7597$

5. Zimmerman CS (2005) Site-specific colpocleisis called superior approach. www.findarticles.com/p/articles/mi_mocyd/is_1_40/ai/ n8968471. Accessed Dec-2009 to Jun-2010

6. Lazarou G, Grioirescu BA, et al (2010) Uterine prolapse: treatment. www.emedcine.medscope.com/../obstetricsandgynecology. Accessed Dec-2009 to Jun-2010

7. Roth TM (2007) Pyometra and recurrent prolapse after Le Fort colpocleisis. Intr Urogynecol J Pelvic Floor Dysfunct 18(6):687688, www.springerlink.com/index/123p2311q40h8p8w.pdf
8. Jelovsek JE, Paraiso MF Surgical procedures for pelvic organ plolapse. www.touchencronology.com/../surgicalprocedurepelvicorganprolapse. Accessed Dec-2009 to Jun-2010

9. Moore RD, Mikloss JR (2001) Repair of vaginal evisceration following colpocleisis utilizing an allogenic dermal graft. Int Eurogynecol J Pelvic Floor Dysfunct 12:215-217, www. miklosandmoore.com/white.pages/uploads/graft/graft_3.pdf (Accessed June 2005)

10. O'Leary AJ, Vyas SK (2004) Le Fort's partial colpocleisis: a review of one surgeon's experience. Gynecological Surgery 1(1):15-19

11. Fitsgerald MP, Richter HE et al (2006) Colpocleisis: a review. Int Eurogynecol J Pelvic Floor Dysfunct 17(3):261-271, www.ncbi. nih.gov/pubmed/15983731

12. Fitsgerald MP (2008) Colpocleisis effective for pelvic organ prolapse. www.findarticles.com/p/articles/mi_hb4365/is_2241/ ai n31151675. Accessed Dec-2009 to Jun-2010

13. Biller D, Davila GW (2004) Choosing the best technique for vaginal vault prolapse. J Fam Prac vol 16 no. 12. www.jfponline. com/pages.asp?aid=3462. Accessed Dec-2009 to Jun-2010

14. Valaitis SR (2010) Obliterative vaginal surgery for the treatment of advanced pelvic prolapse. www.femalepatient.com $/ \mathrm{html} / \mathrm{arc} / . . /$ 030_05 028.asp. Accessed Dec-2009 to Jun-2010

15. Wheeler TL (2005) Regret, satisfaction after partial colpocleisis. www.ncbi.nlm.nih.gov/pubmed/16325617. Accessed Dec-2009 to Jun-2010 